\title{
FLUORID-INDUZIERTE FRAGMENTIERUNG VON ACETYLDIMETHYLPHENYLSILAN
}

\author{
H. ZILCH und R. TACKE* \\ Institut für Anorganische und Analytische Chemie der Technischen Unitersitüt Braunschweig. \\ Hagenring 30, D-3300 Braunschweig (B.R.D.) \\ (Eingegangen den 18. Juni 1986)
}

\section{Summary}

Acetyldimethylphenylsilane (2) reacts with TBAF $\cdot 3 \mathrm{H}_{2} \mathrm{O}$ in THF and with $\mathrm{KF}$ in DMSO $/ \mathrm{H}_{2} \mathrm{O}$, respectively, to give $\left[\left(\mathrm{CH}_{3}\right)_{2} \mathrm{SiO}\right]_{x}$ and 1-Phenylethanol (3) which can be isolated with a nearly quantitative yield. The way 2 reacts with $\mathrm{F}^{-}$contrasts with that of some aroyl- and heteroaroyltrimethylsilanes, described in the literature. A reaction mechanism is discussed which involves among others a 1,2-phenyl shift and a Brook rearrangement.

\section{Zusammenfassung}

Acetyldimethylphenylsilan (2) reagiert bei Raumtemperatur mit TBAF $\cdot 3 \mathrm{H}_{2} \mathrm{O}$ in THF bzw. mit KF in DMSO $/ \mathrm{H}_{2} \mathrm{O}$ zu $\left[\left(\mathrm{CH}_{3}\right)_{2} \mathrm{SiO}\right]_{x}$ und 1-Phenylethanol (3), welches mit praktisch quantitativer Ausbeute isoliert werden kann. Dieses Reaktionsverhalten von 2 gegenüber $\mathrm{F}^{-}$weicht drastisch $\mathrm{ab}$ von dem in der Literatur beschriebenen Verhalten einiger Aroyl- und Heteroaroyltrimethylsilane. Ein Reaktionsmechanismus, der u.a. eine 1,2-Phenylverschiebung und eine BrookUmlagerung beinhaltet, wird zur Diskussion gestellt.

Aroyltrimethylsilane [1,2] und Heteroaroyltrimethylsilane [3] unterliegen in wasserhaltigem Tetrahydrofuran (THF) bei Raumtemperatur einer Fluorid-induzierten $\mathrm{Si}$-C-Spaltung der Si-Acyl-Einheit. Mit dem Ziel, durch eine analoge Reaktion den Acetyl-Rest von Acetyldimethylphenylsilan (2) (erhältlich aus Chlordimethylphenylsilan gemäss Schema 1) abzuspalten, haben wir 2 in THF mit Tetra-n-butylammoniumfluorid-trihydrat (TBAF $\cdot 3 \mathrm{H}_{2} \mathrm{O}$ ) umgesetzt. $\mathrm{Zu}$ unserer Überraschung bildete sich hierbei in einer stark exothermen, sehr raschen Reaktion neben einem nicht näher charakterisierten Siloxan-Gemisch (hauptsächlich Polymere) 1-Phenylethanol (3), das mit praktisch quantitativer Ausbeute (bezogen auf eingesetztes 2) isoliert werden konnte. Das gleiche Ergebnis wurde auch bei der Umsetzung von 2 mit Kaliumfluorid in wasserhaltigem Dimethylsulfoxid (DMSO) erzielt. Weiterführende Untersuchungen [4] haben gezeigt, dass auch Acetyl(t-butyl)methyl- 

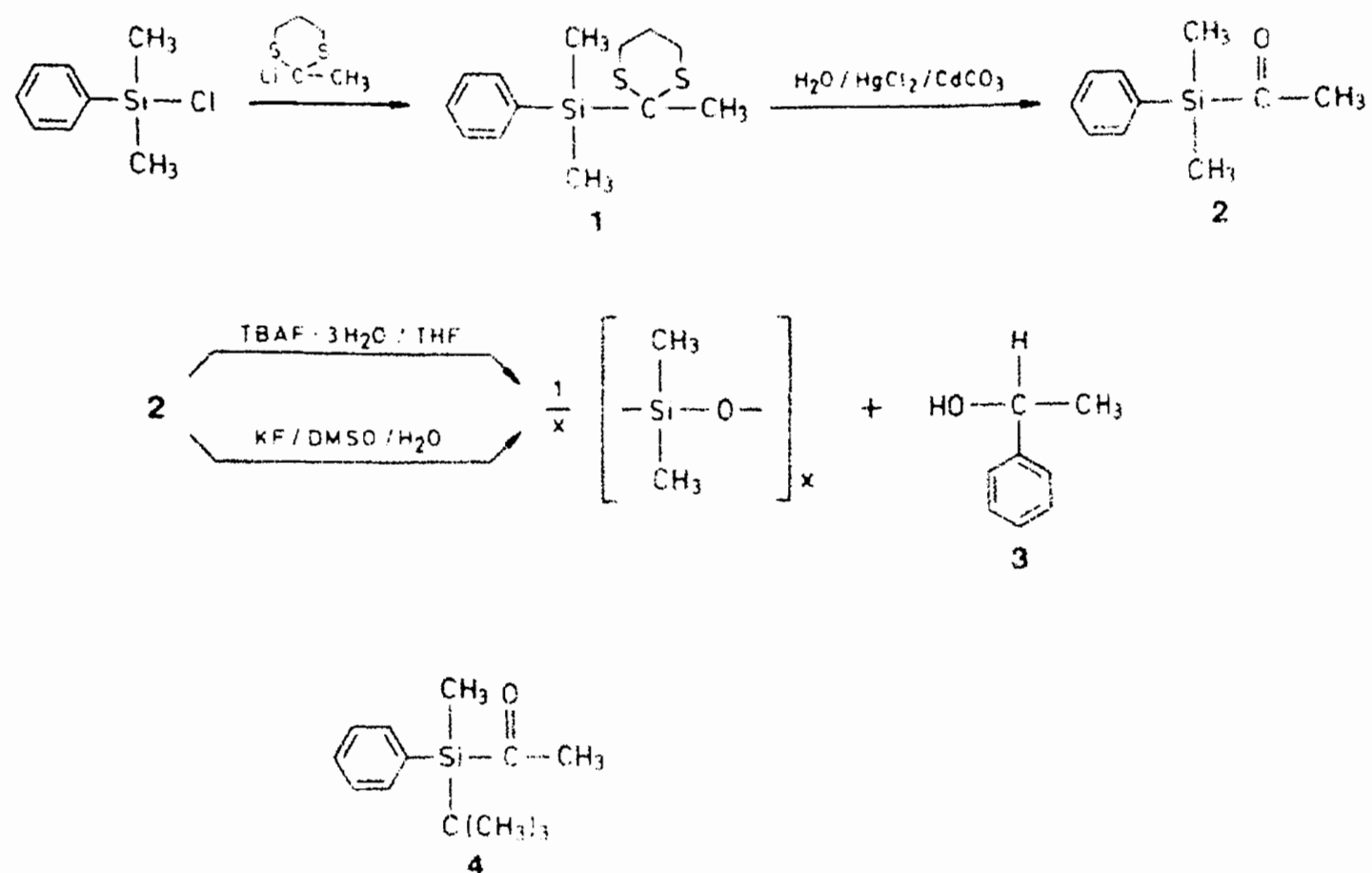

SCHEMA I

phenylsilan (4) analog reagiert: Als Hauptprodukte der Imserzung fon 4 mit TBAF $3 \mathrm{H}_{2} \mathrm{O}$ in THF bei $20^{\circ} \mathrm{C}$ wurden ebenfalls 3 sowie bisher nicht wiaher charakterisierte Siloxane isoliert: als Nebenreaktion wurde hierbe allerdings auch eine "normale" Spaltung der Si...-Bindung der Si Acyl-Einhei beobachtet $\left(\equiv \mathrm{Si}-\mathrm{C}(\mathrm{O}) \mathrm{CH}_{2} \rightarrow \equiv \mathrm{Si}-\mathrm{F}\right.$ ). Die Umsetzung ton 2 mit Fluoridunen verlauft dagegen eindeutig; Produkte, die aus einer "normalen" Si-. C-Spaltung hervogehen würden, konnten nicht nachgewiesen werden ('H-NMR-Kontrolle des Reaktiomsemisches).

Die Fluorid-induzierte Fragmentierung von 2 sowie die analog ahlaufende Hauptreaktion von 4 deuten wir alul der Grundlage einer Kombination cines Fluorid-induzierten 1.2-Phenvl-Verschiebung mit einer Imlagerung wo Brook-Typ. wohei wir uns an die mechanistische Beschreibung [5...7] ciner formal analogen. durch Alkoxidionen induzierten Reaktion von Acylaryldiorganylitanen anlehnen (zur Brook-Umlagerung vgl auch Lit. [8j). Folgender hypothetischer Reaktionsablauf (Schema 2) wird zur Distussion gestellt: Durch Angriff eines Fluoridioms am Siliciumatom von 2 brw. 4 (Ausbildung pentakoordinierter Spezies den Typs A) wird eine 1.2-Phenyl-Verschiebung $10 \mathrm{~m}$ Siliciumatom sum nachbartindigen Carbony. Kohlenstoffatom induzien $(\rightarrow B)$. Hieran schliess sich cinc Umagerung $z$ Carbanionen des Typs C an. deren Protonierung (z.B. Jurch Wasser) a Verbindungen des Typs $\mathbf{D}$ führt, die dann durch hydrolytische Si $(x-$ Spatiung in 3 and die Fluordialkylsilanole E übergehen. Letztere reagieren dann a digomeren hzw. polymeren Siloxanen des Typs $\left(R^{1} R^{2} S i O\right)$, weiter (zum Reaktionsworhalten von Fluordialkylsilanolen vgl. Lit. [9]).

Die in Schema 2 gewählto Beschreibung des Reaktiomsablaufes hat nut formalen Charakter; ob tatsächlich alle der formulierten Zwischenstufen in dieser Form auftreten. muss vorerst offen bleiben. Eine Frklärung dafier Marum sich das Reaktionsverhalten von 2 und 4 so drastisch wo dem der in Lit. [1 ... 3] beschriebenen Aroyl- und Heteroaroyltrimethylsilane unterselesdet wh bistor noch aus. 

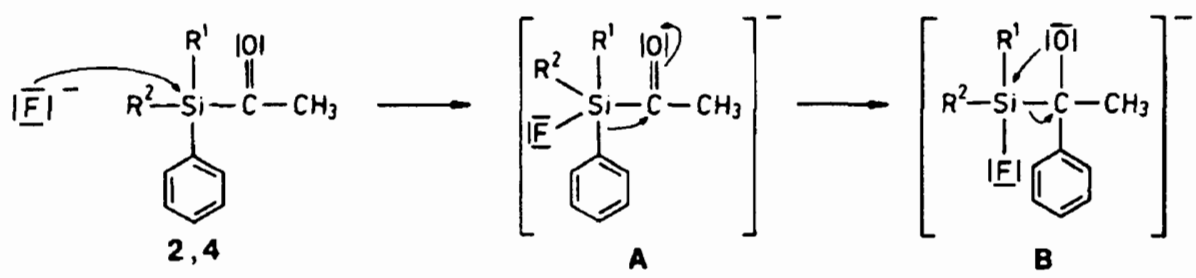

$2: R^{1}=R^{2}=\mathrm{CH}_{3}$

$4: R^{\prime}=\mathrm{CH}_{3}, \mathrm{R}^{2}=\mathrm{C}\left(\mathrm{CH}_{3}\right)_{3}$

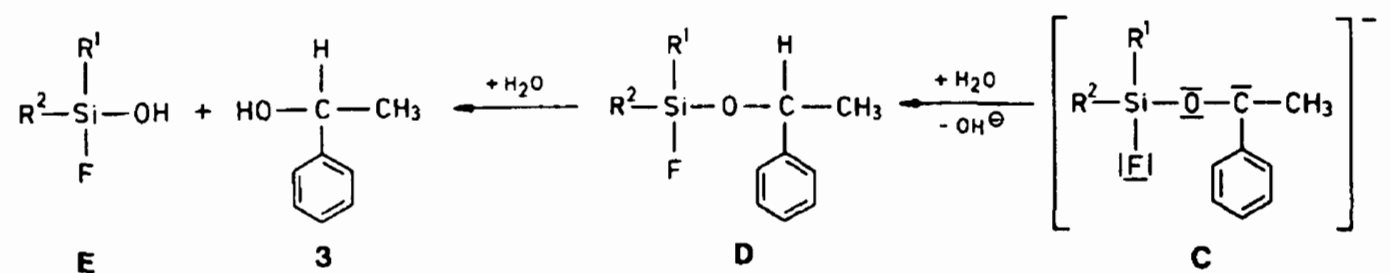

SCHEMA 2

\section{Experimenteller Teil}

Schmelzpunkte (unkorr.): Kofler-Heiztischmikroskop der Fa. Reichert. ${ }^{1} \mathrm{H}$ NMR-Spektren (300.1 MHz, interner Standard $\mathrm{CHCl}_{3}\left(\delta\right.$ 7.25)) und ${ }^{13} \mathrm{C}-\mathrm{NMR}$ Spektren $\left(75.5 \mathrm{MHz}\right.$, interner Standard $\mathrm{CDCl}_{3}(\delta$ 77.0)): AM-300-Gerät der Fa. Bruker. Massenspektren (70 eV; angegeben sind die $\mathrm{m} / \mathrm{z}$-Werte der jeweiligen Molekülionen $M^{+}$sowie der Fragmente mit der höchsten rel. Intensität): CH-7Gerät der Fa. Varian MAT. IR-Spektren: AccuLab-9-Gerät der Fa. Beckman.

\section{Dimethyl(2-methyl-1,3-dithiacyclohexan-2-yl)phenylsilan (1)}

$\mathrm{Zu}$ einer Lösung von $13.43 \mathrm{~g}(0.1 \mathrm{~mol})$ 2-Methyl-1,3-dithiacyclohexan (Darstellung gemäss Lit. [10]) in $400 \mathrm{ml}$ wasserfreiem THF wurden bei $-30^{\circ} \mathrm{C}$ unter Rühren innerhalb von $20 \mathrm{~min} 63 \mathrm{ml}$ einer 15\%igen n-Butyllithium-Lösung in Hexan ( $\cong .104 \mathrm{~mol} \mathrm{BuLi})$ getropft $\left(\mathrm{N}_{2}\right.$-Schutzgasatmosphäre). Man rührte $90 \mathrm{~min}$ bei $-30^{\circ} \mathrm{C}$ weiter, liess innerhalb von ca. $1 \mathrm{~h}$ auf $0^{\circ} \mathrm{C}$ erwärmen, tropfte dann bei dieser Temperatur zu dem so erhaltenen Reaktionsgemisch innerhalb von $30 \mathrm{~min}$ eine Lösung von $17.07 \mathrm{~g}(0.1 \mathrm{~mol})$ Chlordimethylphenylsilan in $100 \mathrm{ml}$ wasserfreiem THF und rührte abschliessend $3 \mathrm{~h}$ bei $0^{\circ} \mathrm{C}$ und $4 \mathrm{~h}$ bei Raumtemperatur. Dann versetzte man das Reaktionsgemisch bei $0^{\circ} \mathrm{C}$ mit $250 \mathrm{ml}$ einer $2 \%$ igen wässerigen $\mathrm{HCl}$-Lösung, trennte nach dem Ausschütteln die etherische Phase ab und trocknete diese über $\mathrm{Na}_{2} \mathrm{SO}_{4}$. Das Lösungsmittel wurde i. Vak. verdampft und der Rückstand bei $-20^{\circ} \mathrm{C}$ aus $\mathrm{n}$-Pentan kristallisiert. Ausb. $16.65 \mathrm{~g}(62 \%)$ weisse Kristalle vom Schmp. $82-83^{\circ} \mathrm{C} .{ }^{1} \mathrm{H}-\mathrm{NMR}\left(\mathrm{CDCl}_{3}\right): \delta 0.52\left(\mathrm{~s}, 6 \mathrm{H}, \mathrm{SiCH}_{3}\right), 1.83\left(\mathrm{~s}, 3 \mathrm{H}, \mathrm{CCH}_{3}\right)$, 1.85-2.1, 2.4-2.5 und 3.0-3.1 (m, 6 H, SCH$\left.{ }_{2} \mathrm{CH}_{2} \mathrm{CH}_{2} \mathrm{~S}\right), 7.3-7.4$ und 7.6-7.7 (m, $\left.5 \mathrm{H}, \mathrm{SiC}_{6} \mathrm{H}_{5}\right) .{ }^{13} \mathrm{C}-\mathrm{NMR}\left(\mathrm{CDCl}_{3}\right): \delta-5.6\left(\mathrm{q}, \mathrm{SiCH}_{3}\right), 23.6\left(\mathrm{t}, \mathrm{CCH}_{2} \mathrm{~S}\right), 24.7(\mathrm{q}$, $\left.\mathrm{CCH}_{3}\right), 25.4\left(\mathrm{t}, \mathrm{CCH} \mathrm{H}_{2} \mathrm{C}\right), 34.4\left(\mathrm{~s}, \mathrm{CCH}_{3}\right), 127.6\left(\mathrm{~d}, \mathrm{C}_{m}\right), 129.6\left(\mathrm{~d}, \mathrm{C}_{p}\right), 134.7\left(\mathrm{~s}, \mathrm{C}_{j}\right)$, $135.0\left(\mathrm{~d}, \mathrm{C}_{o}\right.$ ). MS: $m / z 268\left(77 \%, M^{+}\right), 135\left(100 \%, M^{+}-\mathrm{H}_{3} \mathrm{CCSCH}_{2} \mathrm{CH}_{2} \mathrm{CH}_{2} \mathrm{~S}\right)$. Gef.: C, 58.1; H, 7.4. $\mathrm{C}_{13} \mathrm{H}_{20} \mathrm{~S} 2 \mathrm{Si}(268.5)$ ber.: C, 58.15; H, 7.51\%. 
Acetrldimethylphenlsilan (2)

Fine Lësung von $26.85 \mathrm{~g}$ (i). $\mathrm{mol}) \mathrm{I}$ in $100 \mathrm{ml}$ Aceton wurde bei Ratumtemperittur innerhalh von 15 min 7 cinem (gemisch aus $135.8 \mathrm{~g}(0.5 \mathrm{~mol}) \mathrm{HgCl}, 34.5 \mathrm{~g}(0.2$ mol) $\mathrm{CdCO}, 6.3 \mathrm{ml}$ Wasser, $200 \mathrm{ml}$ Aceton und $600 \mathrm{~m}$ Benzol getropf. Man rührte I h unter Rückfluss. kühlte die schwerbewegliche weisse Suspension auf $10^{\circ} \mathrm{C}$ ab. filtrieste durch cin Papierlatenfilter und wusch den Filtralionsrichstand gründlich

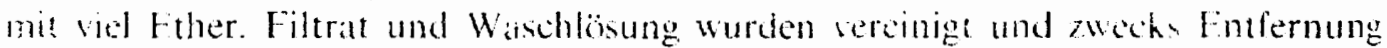
des HgCl, Ünerschusses mehrfith mit einer grösseren Menge itasser baw.

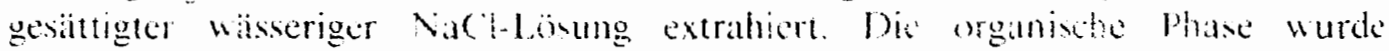
abgetrennt, über $\mathrm{Na}_{\mathrm{S}} \mathrm{SO}_{4}$ getrochnet und i. Vath. Wom lösungmintel hefreit. Dic Vakuumdestillation des Rikchstandes in Kugelrohrverdampler (O)entemperatur

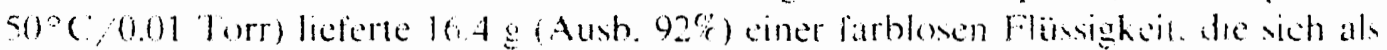
lichtemplindlich und thermsch nur begrenzt bestindig ermies. IR $\left(\mathrm{C}\left(\mathrm{C}_{4}\right)\right.$ : 1650

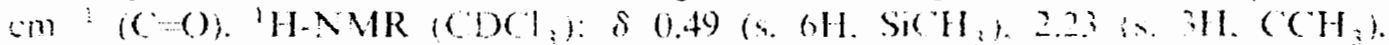

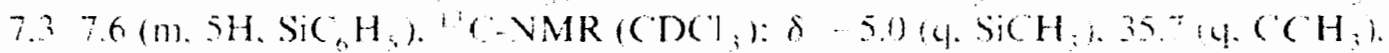

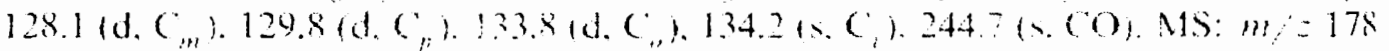

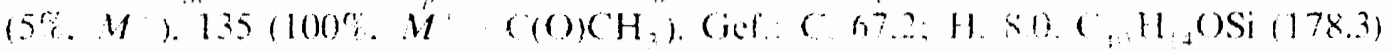
ber.: $(2.67 .36:$ H. $7.91 \%$.

\section{Umicteung tom 2 mit TBAF. 3H.O in THIF}

$Z u$ einer lösung won $1.77 \mathrm{~g}(5.61 \mathrm{mmol}) \mathrm{TBAF} \cdot 3 \mathrm{H}, \mathrm{O}$ in $30 \mathrm{ml}$ THF wurde bei Raumtemperatur unter Rühren innerhalh von $5 \mathrm{~min}$ cine Lönung won $1.00 \mathrm{~g}$ (5.61 mmol) 2 in $10 \mathrm{ml}$ THF getropft (exotherme Reaktion). Anschliessend rührte man 1 It bei Raumtemperatur weiter. verdunnte mit $60 \mathrm{ml}$ Ether. extrahierte acimal mut je $50 \mathrm{~m} /$ Wasser, rocknete die arganische Phase üher $N_{\text {it }}, \mathrm{SO}$, und enternte das lösungsmittel unter verminderlem Druck in Rotationsverdampler. Die Vakummdestillation des Rëckstandes im Kugelrohrverdampler (O)fentemperatur $100^{\circ} \mathrm{C} / 20$ Torr) lieferte $650 \mathrm{mg}$ (Aush $95 \%$ ) ciner larblosen Flïsigken. deren Eigenechaften sich at identisch mit denen einer authentischen prohe val 3. Phenvleshanot eswiesen.

\section{Limsetzung oon 2 mit KF in DMSO}

Eine Lösung von $1.00 \mathrm{~g}(5.67 \mathrm{mmol}) 2 \mathrm{in} 3 \mathrm{ml}$ DMSO wurke hei Raumtemperatur unter Rühren innerhalb von 5 min 70 einer Mischung aus $0.75 \mathrm{gF}, 0.4 \mathrm{ml}$ Wasser und $5 \mathrm{ml}$ DMSO getropft. Das sich stark crwärmende Reak!ionsgemisch wurde 2 h bei Raumtenperalur gerührt und danach mit $70 \mathrm{~m}$ Wasser sersetz. Man extrahierte mit $50 \mathrm{ml}$ Ether. schuittelte die etherische Phase mi $50 \mathrm{mi}$ Wasser aus. trocknete üher $\mathrm{Na}_{2} \mathrm{SO}_{4}$ und befreite unter vernindertem Druck um Liisungsmittel. Die Vakuumdestillation des Rückstandes im Kugelmhrverdampler (O) fentemperatur $100^{\circ} \mathrm{C} / 20$ Torr) lieferte $60.5 \mathrm{mg}$ (Aush. 97\%) einer farblosen Flïsigkeit, deren Figenschaften sich als identisch mit denen einer authentischer Probe wn 1-Phenyethand erwiesen.

\section{Dank}

Diese Arbeit wurde durch Mittel des Fonds der Chemischen Industric sowie durch Chemikalienspenden der Batver AG unterstiatal. 


\section{Literatur}

1 A. Degl'Innocenti, S. Pike, D.R.M. Walton, G. Seconi, A. Ricci und M. Fiorenza, J. Chem. Soc., Chem. Commun., (1980) 1201.

2 D. Schinzer und C.H. Heathcock, Tetrahedron Lett., (1981) 1881.

3 A. Ricci, A. Degl'Innocenti, S. Chimichi, M. Fiorenza und G. Rossini, J. Org. Chem., 50 (1985) 130.

4 R. Tacke, F. Wuttke und H. Zilch, unveröffentlichte Ergebnisse.

5 A.G. Brook, Adv. Organomet. Chem., 7 (1968) 95.

6 A.G. Brook, T.J.D. Vandersar und W. Limburg, Can. J. Chem., 56 (1978) 2758.

7 A.G. Brook und A.R. Bassindale, in P. de Mayo (Hrsg.), Rearrangements in Ground and Excited States, Vol. 2. Academic Press, New York/London/Sydney/San Francisco, 1980, S. 149-227.

8 A.G. Brook, J. Organomet. Chem., 300 (1986) 21.

9 U. Klingebiel, Chem. Ber., 114 (1981) 3366.

10 E.J. Corey und B.W. Erickson. J. Org. Chem., 36 (1971) 3553. 